\title{
Identification of maximal running intensities during elite hurling match-play
}

\author{
Damien Young \\ Technological University of the Shannon: Midlands Midwest \\ Shane Malone \\ Technological University Dublin, Shane.Malone@TUDublin.ie \\ Marco Beato \\ Suffolk University
}

See next page for additional authors

Follow this and additional works at: https://arrow.tudublin.ie/ittsciart

Part of the Sports Sciences Commons

\section{Recommended Citation}

Young D, Malone S, Beato M, Mourot L, Coratella G. Identification of Maximal Running Intensities During Elite Hurling Match-Play. J Strength Cond Res. 2020 Sep;34(9):2608-2617. doi: 10.1519/

JSC.0000000000002674. PMID: 29939899.

This Article is brought to you for free and open access by the School of Science and Computing at ARROW@TU Dublin. It has been accepted for inclusion in Articles by an authorized administrator of ARROW@TU Dublin. For more information, please contact arrow.admin@tudublin.ie, aisling.coyne@tudublin.ie, gerard.connolly@tudublin.ie.

Funder: French Ministry of National Education \& Ministry of Research and of Technology; Tomsk Polytechnic University Competitiveness Enhancement Program

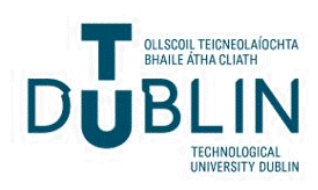


Authors

Damien Young, Shane Malone, Marco Beato, Laurent Mourot, and Giuseppe Coratella

This article is available at ARROW@TU Dublin: https://arrow.tudublin.ie/ittsciart/99 
See discussions, stats, and author profiles for this publication at: https://www.researchgate.net/publication/325935493

\section{Identification of Maximal Running Intensities During Elite Hurling Match-Play}

Article in The Journal of Strength and Conditioning Research · June 2018

DOI: $10.1519 /$ JSC. 0000000000002674

CITATIONS

20

5 authors, including:

Damien Young

Technological University of the Shannon: Midlands Midwest

20 PUBLICATIONS 131 CITATIONS

SEE PROFILE

Marco Beato

University of Suffolk

106 PUBLICATIONS 1,298 CITATIONS

SEE PROFILE

Some of the authors of this publication are also working on these related projects:

Circadian Rhythm of Heart Hemodynamic and Autonomic Variables View project

Environnement and exercise: Friend or Foe? View project
READS

1,098

13 Technological University Dublin - Tallaght Campus

65 PUBLICATIONS 1,494 CITATIONS

SEE PROFILE

Laurent Mourot

University Bourgogne Franche-Comté

170 PUBLICATIONS 2,609 CITATIONS

SEE PROFILE 


\title{
Identification of Maximal Running Intensities During Elite Hurling Match-Play
}

\author{
Damien Young, ${ }^{1}$ Shane Malone, ${ }^{2,3}$ Marco Beato, ${ }^{4}$ Laurent Mourot, ${ }^{1,5,6}$ and \\ Giuseppe CoRatella ${ }^{7}$ \\ ${ }^{1}$ Research Unit EA3920 Prognostic Markers and Regulatory Factors of Cardiovascular Diseases and Exercise Performance, \\ Exercise Performance Health, Innovation Platform, University of Bourgogne Franche-Comte, Besançon, France; ${ }^{2}$ Gaelic Sports \\ Research Center, Institute of Technology Tallaght, Dublin, Ireland; ${ }^{3}$ The Tom Reilly Building, Research Institute for Sport and \\ Exercise Sciences, Liverpool John Moores University, Liverpool, United Kingdom; ${ }^{4}$ Faculty of Health and Science, Department \\ of Science and Technology, University of Suffolk, Ipswich, United Kingdom; ${ }^{5}$ EA3920 Prognostic Factors and Regulatory \\ Factors of Cardiac and Vascular Pathologies, Exercise Performance Health Innovation (EPHI), University of Bourgogne \\ Franche-Comté, Besançon, France; ${ }^{6}$ Tomsk Polytechnic University, Tomsk, Russia; and ${ }^{7}$ Department of Biomedical Sciences for \\ Health, University of Milan, Italy
}

\begin{abstract}
Young, D, Malone, S, Beato, M, Mourot, L, and Coratella, G. lidentification of maximal running intensities during elite hurling match-play. J Strength Cond Res XX(X): 000-000, 2018-The current study aimed to describe the duration-specific running intensities of elite hurling players during competition with respect to position using a rolling average method. Global positioning systems (10-Hz Viper; STATSport, Viper, Newry, Northern Ireland) were used to collect data from 36 elite hurling players across 2 seasons. Players were categorized according to playing positions (full-backs, half-backs, midfielders, half-forwards, and full-forwards). A total of 230 full match samples were obtained from 22 competitive games for analysis. The velocity-time curve was analyzed using a rolling average method, in which the maximum relative total distance (TD; $\left.\mathrm{m} \cdot \mathrm{min}^{-1}\right)$, high-speed running distance $\left(\mathrm{HSR} ; \mathrm{m} \cdot \mathrm{min}^{-1}\right.$ ), and sprint distance (SD; $\mathrm{m} \cdot \mathrm{min}^{-1}$ ) intensities were calculated across 10 different rolling time durations (1-10 minutes) within each game. There were large to very large (effect sizes [ES] = 0.66-4.33) differences between 1 minute rolling averages and all other durations for TD, HSR, and SD. However, pairwise comparisons between 6 and 10 minutes for TD, HSR, and SD were smaller and more variable $(E S=0.07$, trivial to $E S=0.85$, moderate). Half-backs, midfielders, and half-forwards achieved a higher maximal relative TD and HSR in all duration-specific fields when compared with full-backs and full-forwards. No positional difference was observed in 1- and 2-minute durations for SD. Because the rolling average duration increased
\end{abstract}

Address correspondence to Damien Young, damien.young@hotmail.com. 00(00)/1-10

Journal of Strength and Conditioning Research

(C) 2018 National Strength and Conditioning Association the maximum TD, HSR and SD running intensities decreased across all positions. These data provide knowledge of the peak running intensities of elite hurling competition and can be used to design training activities to sufficiently prepare players for these "worst-case scenarios."

KEY WoRDS GPS, rolling average, high-speed running, worse-case running, sprint distance

\section{INTRODUCTION}

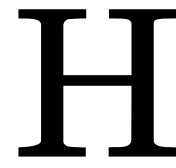

urling is an intermittent stick and ball invasion team sport and one of the national sports of Ireland. It is a physically demanding and highly skillful game with periods of high-intensity efforts similar to other team sports $(7,26,27)$. The game is 70 minutes ( 35 minutes per half) in duration and is played on a pitch $140 \mathrm{~m}$ long and $90 \mathrm{~m}$ wide. Two teams of 15 players (1 goalkeeper and 14 outfield players) contest for possession, and the aim is to outscore the opposing team by striking the ball through their goalpost, under or over the crossbar earning 3 points (goal) and 1 point, respectively (24). Players' physical, tactical, and technical roles differ between the 5 distinctive positions (full-backs, half-backs, midfielders, half-forwards, and full-forwards) $(7,26,27)$. Ten elite teams each representing a county compete for Provincial and All-Ireland senior championship, which attract large attendances of over 80,000 spectators for the final (24).

Recently, there has been an increase in the utilization of global positioning system (GPS) technology to track players' training and match-play running demands $(6,7,17)$. The running performance of elite senior hurling match-play was quantified using GPS (7). Results have shown that players cover a total distance (TD), high-speed running distance 
(HSR), and sprint distance (SD) of approximately 7,600, 780, $500-\mathrm{m}$, respectively, during the 70 minutes of competitive play (7). Relatively, players cover $109 \mathrm{~m} \cdot \mathrm{min}^{-1}$, with 16 and $5 \mathrm{~m} \cdot \mathrm{min}^{-1}$ spent at HSR and SD, respectively (7). Most HSR efforts occur close to the hurling ball and may determine the outcome of crucial events in the game (24). Furthermore, there were small to moderate differences in TD, HSR, and SD between positions, with midfielders being the highest performers when compared with the remaining 4 positions (7). Full-backs undertake the least TD and HSR (7). Similar to other team sports $(9,19,21)$, a distinct positional profile for running performance exists during hurling match-play, which coaches must consider when selecting specific training methodologies for competition.

Temporal decrements in running performance exist within team sports, with these being related to transient fatigue experienced throughout a match (23). In professional rugby union, players covered the greatest relative distance during the first 10-minute period of each half of the play (12). In soccer, players were shown to cover greater distances in the first 15 minutes $(1,919 \pm 125 \mathrm{~m})$ of the match compared with the last 15 minutes $(1,775 \pm 158 \mathrm{~m})$ (5). Similarly, in hurling, players' running performance deteriorated across halves and quarters of the play (7). Data presented per predefined periods provide an understanding as to the temporal change in running performance. These global relative running distances fail to fully account for the most intense periods of play or "worst-case scenario" period that occurs during match-play. Given that relative distances are used to determine training activities, these activities may be under preparing players to complete these "worst-case scenarios" of match-play (9). Previously, quantifying the match-play demands relative to match duration underestimates the most intense running performance periods during team invasion sports $(9,21)$. The use of a rolling average method, in which distance covered in set intervals from every time point is sampled during each half, could be a more appropriate method when quantifying the running intensity periods in team sports (9). Results from professional soccer showed that the peak predefined 5 -minute block $(142 \pm 24 \mathrm{~m})$ underestimated the true peak running intensity by up to $25 \%$ when compared with a 5-minute rolling average (177 $\pm 91 \mathrm{~m}$ ) method (25). These running performances substantially increase as the length of the rolling average duration decreases $(9,21)$.

In senior hurling, significant differences between playing positions were found in TD, HSR, and SD. Midfielders covered greater distances in TD and HSR compared with all other positions (7). Furthermore, decrements in HSR have been observed between positions, with the half-forwards experiencing the highest drop-off and midfielders and fullbacks experiencing the lowest drop-off (7). However, it is unclear if there are any positional differences in the relative $\left(\mathrm{m} \cdot \mathrm{min}^{-1}\right)$ running intensities in hurling. Recently, results in Gaelic football showed that players covered greater relative distances (full-backs: $194 \mathrm{~m} \cdot \mathrm{min}^{-1}$, half-backs: 242 $\mathrm{m} \cdot \mathrm{min}^{-1}$, midfielders: $255 \mathrm{~m} \cdot \mathrm{min}^{-1}$, half-forwards: 241 $\mathrm{m} \cdot \mathrm{min}^{-1}$, and full-forwards: $196 \mathrm{~m} \cdot \mathrm{min}^{-1}$ ) using the rolling average method compared with the distance per min covered $\left(116-151 \mathrm{~m} \cdot \mathrm{min}^{-1}\right)$ relative to the match duration method $(19,20)$.

Recently, some studies have attempted to describe the physical demands of hurling $(7,26,27)$. However, to date no research has reported the specific "worse-case" running performance of hurling players during match-play. Without this information, coaches may be under preparing players for the competitive demands of match-play. Therefore, the aim of the current study was to describe the duration-specific running intensities of elite hurling players during competition with respect to position using a rolling average method. It was hypothesized that the peak relative running periods will be higher than that previously reported and would be position specific.

\section{Methods}

\section{Experimental Approach to the Problem}

The current observational study was designed to examine the duration-specific running intensities of elite hurling players during competition with respect to position across predefined time periods (1-10 minutes) using a rolling average method. Thirty-six $(n=36)$ elite hurling players playing at the highest level (Provincial and All-Ireland Championship) were recruited and analyzed across 2 full competition seasons (February 2016-August 2017) resulting in 230 individual samples being collected $(n=230)$. Game data were collected across 22 games during the 2016 and 2017 seasons. Data were included only if a full match (70 minutes) was completed. The players were classified according to their playing position during each match resulting in the following number of data sets per position: full-backs: $n=48$, half-backs: $n=48$, midfielders: $n=38$, half-forwards: $n=48$, and full-forwards: $n=48$. All competitive matches took place between 14.00 and 18.00 hours. Temperatures ranged from 11 to $21^{\circ} \mathrm{C}$. Global positioning systems were used to quantify players' running performance during competitive games. After match-play, a customized spreadsheet was used to specifically allow for the computation of a rolling average for each player's relative TD covered $\left(\mathrm{m} \cdot \mathrm{min}^{-1}\right)$, HSR $\left(\mathrm{m} \cdot \mathrm{min}^{-1} ; \geq 17-22 \mathrm{~km} \cdot \mathrm{h}^{-1}\right)$, and SD $\left(\mathrm{m} \cdot \mathrm{min}^{-1} ;>22 \mathrm{~km} \cdot \mathrm{h}^{-1}\right)(21)$. To assist in the development of velocity-based movement indicators, rolling moving averages were calculated across 10 different durations $(1,2,3,4,5,6,7,8,9$, and 10 minutes) for each player across each match, with the maximum value for each specific duration recorded $(9,21)$.

\section{Subjects}

Thirty-six $(n=36)$ elite male hurlers (mean $\pm S D$, age: $27 \pm$ 4 years; mean $\pm S D$, height: $181 \pm 5 \mathrm{~cm}$; mean $\pm S D$, mass: $86 \pm 4 \mathrm{~kg}$ ) volunteered for the current investigation over 2 
TABLE 1. Comparisons between maximal relative total distance $\left(\mathrm{m} \cdot \mathrm{min}^{-1}\right)$ for rolling averages of different durations (mean \pm 90 confidence intervals).*

\begin{tabular}{|c|c|c|c|c|c|c|c|c|c|}
\hline & 1 & 2 & 3 & 4 & 5 & 6 & 7 & 8 & 9 \\
\hline 2 & $\begin{array}{c}25 \pm 27 \\
\text { Likely } \\
\text { Large } \uparrow\end{array}$ & & & & & & & & \\
\hline \multirow[t]{2}{*}{3} & $36 \pm 26$ & $11 \pm 23$ & & & & & & & \\
\hline & $\begin{array}{l}\text { Very likely } \\
\text { Large } \uparrow\end{array}$ & $\begin{array}{c}\text { Possibly } \\
\text { Moderate } \uparrow\end{array}$ & & & & & & & \\
\hline \multirow[t]{3}{*}{4} & $42 \pm 26$ & $17 \pm 23$ & $6 \pm 21$ & & & & & & \\
\hline & Very likely & Possibly & Possibly & & & & & & \\
\hline & Very large $\uparrow$ & Moderate $\uparrow$ & Small $\uparrow$ & & & & & & \\
\hline \multirow[t]{3}{*}{5} & $47 \pm 26$ & $22 \pm 23$ & $11 \pm 21$ & $5 \pm 21$ & & & & & \\
\hline & Very likely & Likely & Possibly & Possibly & & & & & \\
\hline & Very large $\uparrow$ & Large $\uparrow$ & Moderate $\uparrow$ & Small & & & & & \\
\hline \multirow[t]{2}{*}{6} & $\begin{array}{l}50 \pm 25 \\
\text { Most likely }\end{array}$ & $\begin{array}{c}25 \pm 23 \\
\text { Likely }\end{array}$ & $\begin{array}{l}14 \pm 20 \\
\text { Possibly }\end{array}$ & $\begin{array}{l}8 \pm 20 \\
\text { Possibly }\end{array}$ & $\begin{array}{l}3 \pm 20 \\
\text { Possibly }\end{array}$ & & & & \\
\hline & Very large $\uparrow$ & Large $\uparrow$ & Moderate $\uparrow$ & Small $\uparrow$ & Small $\uparrow$ & & & & \\
\hline \multirow[t]{2}{*}{7} & $\begin{array}{c}52 \pm 25 \\
\text { Most likely }\end{array}$ & $\begin{array}{c}27 \pm 23 \\
\text { Likely }\end{array}$ & $\begin{array}{l}16 \pm 20 \\
\text { Possibly }\end{array}$ & $\begin{array}{l}10 \pm 20 \\
\text { Possibly }\end{array}$ & $\begin{array}{l}5 \pm 20 \\
\text { Possibly }\end{array}$ & $\begin{array}{l}2 \pm 20 \\
\text { Unlikely }\end{array}$ & & & \\
\hline & Very large $\uparrow$ & Large $\uparrow$ & Moderate $\uparrow$ & Moderate $\uparrow$ & Small $\uparrow$ & Trivial $\uparrow$ & & & \\
\hline \multirow[t]{2}{*}{8} & $\begin{array}{c}54 \pm 25 \\
\text { Most likely }\end{array}$ & $\begin{array}{c}29 \pm 23 \\
\text { Likely }\end{array}$ & $\begin{array}{l}18 \pm 20 \\
\text { Possibly }\end{array}$ & $\begin{array}{l}12 \pm 20 \\
\text { Possibly }\end{array}$ & $\begin{array}{c}7 \pm 20 \\
\text { Possibly }\end{array}$ & $\begin{array}{l}4 \pm 20 \\
\text { Possibly }\end{array}$ & $\begin{array}{l}2 \pm 20 \\
\text { Unlikely }\end{array}$ & & \\
\hline & Very large $\uparrow$ & Large $\uparrow$ & Large $\uparrow$ & Moderate $\uparrow$ & Small $\uparrow$ & Small $\uparrow$ & Trivial $\uparrow$ & & \\
\hline \multirow[t]{2}{*}{9} & $\begin{array}{c}56 \pm 25 \\
\text { Most likely }\end{array}$ & $\begin{array}{c}31 \pm 23 \\
\text { Likely }\end{array}$ & $\begin{array}{c}20 \pm 20 \\
\text { Likely }\end{array}$ & $\begin{array}{l}14 \pm 20 \\
\text { Possibly }\end{array}$ & $\begin{array}{l}9 \pm 20 \\
\text { Possibly }\end{array}$ & $\begin{array}{l}6 \pm 20 \\
\text { Possibly }\end{array}$ & $\begin{array}{l}4 \pm 20 \\
\text { Possibly }\end{array}$ & $\begin{array}{l}2 \pm 20 \\
\text { Unlikely }\end{array}$ & \\
\hline & Very large $\uparrow$ & Large $\uparrow$ & Large $\uparrow$ & Moderate $\uparrow$ & Moderate $\uparrow$ & Small $\uparrow$ & Small $\uparrow$ & Trivial $\uparrow$ & \\
\hline \multirow[t]{2}{*}{10} & $\begin{array}{c}57 \pm 24 \\
\text { Most likely }\end{array}$ & $\begin{array}{l}32 \pm 22 \\
\text { Very likely }\end{array}$ & $\begin{array}{c}21 \pm 20 \\
\text { Likely }\end{array}$ & $\begin{array}{c}15 \pm 6 \\
\text { Possibly }\end{array}$ & $\begin{array}{l}10 \pm 20 \\
\text { Possibly }\end{array}$ & $\begin{array}{l}7 \pm 19 \\
\text { Possibly }\end{array}$ & $\begin{array}{l}5 \pm 19 \\
\text { Possibly }\end{array}$ & $\begin{array}{l}3 \pm 19 \\
\text { Possibly }\end{array}$ & $\begin{array}{l}1 \pm 19 \\
\text { Unlikely }\end{array}$ \\
\hline & Very large $\uparrow$ & Large $\uparrow$ & Large $\uparrow$ & Moderate $\uparrow$ & Moderate $\uparrow$ & Small $\uparrow$ & Small $\uparrow$ & Small $\uparrow$ & Trivial $\uparrow$ \\
\hline
\end{tabular}

*Data are presented as mean $90 \% \mathrm{Cl}$, likelihood of effect, size and direction of effect $\uparrow$ or $\downarrow$.

seasons. The players were selected from members of an elite senior hurling squad who were deemed the best players in the county at the time of data collection (18). Only those who were free from injury and illness were eligible to partake in the study. The subjects were informed of the purpose, benefits, and potential risks of the study. Written informed consent and medical declaration were obtained from all participants. Finally, the University Bourgogne Franche-Comté Ethics Committee approved all procedures, and the study was conducted according to the Declaration of Helsinki (1975) for studies involving human subjects.

\section{Procedures}

The running performance of players during match-play was recorded using $10-\mathrm{Hz}$ GPS units and $100-\mathrm{Hz}$ triaxial accelerometer (STATSports, Viper, Newry, Northern Ireland: Firmware 2.28) $(1,3,4)$. The validity and reliability of these GPS units were previously established (2). The GPS unit (dimensions $86 \times 33 \times 14 \mathrm{~mm}$, mass $50 \mathrm{~g}$ ) was placed within a pouch between the player's shoulder blades (upper thoracic spine) in a sports vest and worn under the playing jersey. Global positioning system activation and satellite lock were established
15 minutes before warm-up commencement (16). The participants were familiarized with the GPS technology during team training sessions before data collection. To minimize the effect of the inter-unit error, each player was allocated the same unit for the duration of the investigation (11).

After the completion of each match, GPS data were downloaded to a computer through the bespoke STATSport analysis software (STATSport Viper Firmware 2.28) to be stored and analyzed. Each file was trimmed so that data recorded only when the player was on the field were included for further analysis. The proprietary software provided instantaneous raw velocity data at 0.10 -second intervals, which was then exported and placed into a customized Microsoft Excel spreadsheet (Microsoft, Redmond, WA, USA). The spreadsheet allowed analysis of the relative distance covered $\left(\mathrm{m} \cdot \mathrm{min}^{-1}\right)$ in the following categories: TD $\left(\mathrm{m} \cdot \mathrm{min}^{-1}\right)$, high-speed distance $\left(\mathrm{m} \cdot \mathrm{min}^{-1}, 17-22 \mathrm{~km} \cdot \mathrm{h}^{-1}\right)$, and $\mathrm{SD}\left(\mathrm{m} \cdot \mathrm{min}^{-1} ;>22 \mathrm{~km} \cdot \mathrm{h}^{-1}\right)(26)$. To best allow for the development of a velocity-based running performance curve, rolling averages were analyzed across 10 specific durations (1-10 minutes) for each player, with a maximum value for each specific duration recorded. 
TABle 2. Comparisons between maximal high-speed running distance $\left(\mathrm{m} \cdot \mathrm{min}^{-1}\right)$ for rolling averages of different durations (mean \pm 90 confidence intervals). *

\begin{tabular}{|c|c|c|c|c|c|c|c|c|c|}
\hline & 1 & 2 & 3 & 4 & 5 & 6 & 7 & 8 & 9 \\
\hline 2 & $\begin{array}{c}15 \pm 16 \\
\text { Likely } \\
\text { Large } \uparrow\end{array}$ & & & & & & & & \\
\hline 3 & $\begin{array}{c}22 \pm 15 \\
\text { Very likely } \\
\text { Very large } \uparrow\end{array}$ & $\begin{array}{c}7 \pm 11 \\
\text { Possibly } \\
\text { Moderate } \uparrow\end{array}$ & & & & & & & \\
\hline 4 & $\begin{array}{c}26 \pm 14 \\
\text { Very likely } \\
\text { Very large } \uparrow\end{array}$ & $\begin{array}{c}11 \pm 11 \\
\text { Likely } \\
\text { Large } \uparrow\end{array}$ & $\begin{array}{c}4 \pm 9 \\
\text { Possibly } \\
\text { Moderate } \uparrow\end{array}$ & & & & & & \\
\hline 5 & $\begin{array}{c}28 \pm 14 \\
\text { Very likely } \\
\text { Very large } \uparrow\end{array}$ & $\begin{array}{l}13 \pm 11 \\
\text { Likely } \\
\text { Large } \uparrow\end{array}$ & $\begin{array}{c}6 \pm 9 \\
\text { Possibly } \\
\text { Moderate } \uparrow\end{array}$ & $\begin{array}{l}2 \pm 8 \\
\text { Unlikely } \\
\text { Small } \uparrow\end{array}$ & & & & & \\
\hline 6 & $\begin{array}{c}29 \pm 14 \\
\text { Most likely } \\
\text { Very large } \uparrow\end{array}$ & $\begin{array}{l}14 \pm 10 \\
\text { Likely } \\
\text { Large } \uparrow\end{array}$ & $\begin{array}{c}7 \pm 9 \\
\text { Possibly } \\
\text { Moderate } \uparrow\end{array}$ & $\begin{array}{l}3 \pm 8 \\
\text { Possibly } \\
\text { Small } \uparrow\end{array}$ & $\begin{array}{l}1 \pm 8 \\
\text { Unlikely } \\
\text { Trivial } \uparrow\end{array}$ & & & & \\
\hline 7 & $\begin{array}{c}31 \pm 14 \\
\text { Most likely } \\
\text { Very large } \uparrow\end{array}$ & $\begin{array}{c}16 \pm 10 \\
\text { Likely } \\
\text { Very large } \uparrow\end{array}$ & $\begin{array}{c}9 \pm 9 \\
\text { Possibly } \\
\text { Large } \uparrow\end{array}$ & $\begin{array}{c}5 \pm 8 \\
\text { Possibly } \\
\text { Moderate } \uparrow\end{array}$ & $\begin{array}{c}3 \pm 8 \\
\text { Possibly } \\
\text { Small } \uparrow\end{array}$ & $\begin{array}{l}2 \pm 7 \\
\text { Unlikely } \\
\text { Small } \uparrow\end{array}$ & & & \\
\hline 8 & $\begin{array}{c}31 \pm 14 \\
\text { Most likely } \\
\text { Very large } \uparrow\end{array}$ & $\begin{array}{c}16 \pm 10 \\
\text { Likely } \\
\text { Very large } \uparrow\end{array}$ & $\begin{array}{c}9 \pm 9 \\
\text { Possibly } \\
\text { Large } \uparrow\end{array}$ & $\begin{array}{c}5 \pm 8 \\
\text { Possibly } \\
\text { Moderate } \uparrow\end{array}$ & $\begin{array}{c}3 \pm 8 \\
\text { Possibly } \\
\text { Small } \uparrow\end{array}$ & $\begin{array}{l}2 \pm 7 \\
\text { Unlikely } \\
\text { Small } \uparrow\end{array}$ & $\begin{array}{c}0 \pm 7 \\
\text { Unlikely } \\
\text { Trivial } \uparrow\end{array}$ & & \\
\hline 9 & $\begin{array}{c}32 \pm 14 \\
\text { Most likely } \\
\text { Very large } \uparrow\end{array}$ & $\begin{array}{c}17 \pm 10 \\
\text { Very likely } \\
\text { Very large } \uparrow\end{array}$ & $\begin{array}{l}10 \pm 9 \\
\text { Likely } \\
\text { Large } \uparrow\end{array}$ & $\begin{array}{c}6 \pm 8 \\
\text { Possibly } \\
\text { Moderate } \uparrow\end{array}$ & $\begin{array}{c}4 \pm 8 \\
\text { Possibly } \\
\text { Moderate } \uparrow\end{array}$ & $\begin{array}{l}3 \pm 7 \\
\text { Unlikely } \\
\text { Small } \uparrow\end{array}$ & $\begin{array}{c}1 \pm 7 \\
\text { Unlikely } \\
\text { Trivial } \uparrow\end{array}$ & $\begin{array}{c}1 \pm 7 \\
\text { Unlikely } \\
\text { Trivial } \uparrow\end{array}$ & \\
\hline 10 & $\begin{array}{c}33 \pm 14 \\
\text { Most likely } \\
\text { Very large } \uparrow\end{array}$ & $\begin{array}{c}18 \pm 10 \\
\text { Very likely } \\
\text { Very large } \uparrow\end{array}$ & $\begin{array}{l}11 \pm 9 \\
\text { Likely } \\
\text { Large } \uparrow\end{array}$ & $\begin{array}{c}7 \pm 8 \\
\text { Possibly } \\
\text { Large } \uparrow\end{array}$ & $\begin{array}{c}5 \pm 8 \\
\text { Possibly } \\
\text { Moderate } \uparrow\end{array}$ & $\begin{array}{c}4 \pm 7 \\
\text { Possibly } \\
\text { Moderate } \uparrow\end{array}$ & $\begin{array}{l}2 \pm 7 \\
\text { Unlikely } \\
\text { Small } \uparrow\end{array}$ & $\begin{array}{l}2 \pm 7 \\
\text { Unlikely } \\
\text { Small } \uparrow\end{array}$ & $\begin{array}{c}1 \pm 7 \\
\text { Unlikely } \\
\text { Trivial } \uparrow\end{array}$ \\
\hline
\end{tabular}

*Data are presented as mean $90 \% \mathrm{Cl}$, likelihood of effect, size and direction of effect $\uparrow$ or $\downarrow$.

\section{Statistical Analyses}

Data are presented as mean $\pm S D$ and $90 \%$ confidence intervals. Descriptive analysis and assumptions of normality were verified before parametric statistical analysis. Multivariate analysis of variance was used to compare differences in maximal running performance rolling averages between positions and time duration. A Bonferroni post hoc test was used to analyze significant between-position differences. Statistical significance was set at $\alpha \leq 0.05$. Cohen's effect size (d) was used to describe the differences in running performance between rolling average lengths. Standardized effect sizes (ES) were calculated with $d<0.20,0.20-0.59,0.60$ $1.19,1.20-1.99$, and $\geq 2.00$ and interpreted as follows: trivial, small, moderate, large, and very large differences, respectively, as recommended by Hopkins (10). Furthermore, a customized spreadsheet (Microsoft Excel; Microsoft) was used to calculate the chance that the observed difference between rolling averages was practically significant (10), using thresholds of $10 \mathrm{~m} \cdot \mathrm{min}^{-1}$ (TD) (8), $6 \mathrm{~m} \cdot \mathrm{min}^{-1}$ (HSR), and 2 $\mathrm{m} \cdot \mathrm{min}^{-1}(\mathrm{SD})(21)$. These thresholds represent the smallest worthwhile change in the specific variable of analysis that would practically have an application in the prescription and monitoring of hurling training $(8,9)$. Quantitative chances of real differences in variables were assessed qualitatively as follows: $<0.5 \%$, most unlikely; $0.5-5 \%$, very unlikely; $5.1-$ 25\%, unlikely; $25.1-75 \%$, possibly; $75.1-95 \%$, likely; $95.1-$ $99.5 \%$, very likely; and $>99.5 \%$, most likely (10). Statistical analysis was performed using SPSS version 22.0 (IBM Corp., Armonk, NY, USA).

\section{Results}

Independent of the position, the average match-play demands of elite hurling were 7,358 $\pm 1,085,759 \pm 206$, and $486 \pm 127 \mathrm{~m}$ for TD, HSR, and SD, respectively. Furthermore, the maximal relative demands for TD, HSR, and SD were $184 \pm 21,51 \pm 13$, and $42 \pm 10 \mathrm{~m} \cdot \mathrm{min}^{-1}$, respectively.

\section{Running Performance Based on Rolling Average Duration}

The pairwise comparisons between rolling average durations and running performance variables for relative TD are displayed in Table 1 . The difference in TD between the highest 4 rolling averages $(6-10$ minutes $)$ was trivial

4 Journal of Strength and Conditioning Research 
TABLE 3. Comparisons between maximal sprint distance $\left(\mathrm{m} \cdot \mathrm{min}^{-1}\right)$ for rolling averages of different durations (mean \pm 90 confidence intervals).*

\begin{tabular}{|c|c|c|c|c|c|c|c|c|c|}
\hline & 1 & 2 & 3 & 4 & 5 & 6 & 7 & 8 & 9 \\
\hline 2 & $\begin{array}{c}16 \pm 13 \\
\text { Very likely } \\
\text { Large } \uparrow\end{array}$ & & & & & & & & \\
\hline 3 & $\begin{array}{c}22 \pm 12 \\
\text { Most likely } \\
\text { Very large } \uparrow\end{array}$ & $\begin{array}{c}6 \pm 10 \\
\text { Possibly } \\
\text { Moderate } \uparrow\end{array}$ & & & & & & & \\
\hline 4 & $\begin{array}{c}25 \pm 11 \\
\text { Most likely } \\
\text { Very large } \uparrow\end{array}$ & $\begin{array}{c}9 \pm 9 \\
\text { Likely } \\
\text { Large } \uparrow\end{array}$ & $\begin{array}{c}3 \pm 8 \\
\text { Possibly } \\
\text { Small } \uparrow\end{array}$ & & & & & & \\
\hline 5 & $\begin{array}{c}27 \pm 11 \\
\text { Most likely } \\
\text { Very large } \uparrow\end{array}$ & $\begin{array}{c}11 \pm 9 \\
\text { Very likely } \\
\text { Large } \uparrow\end{array}$ & $\begin{array}{c}5 \pm 7 \\
\text { Likely } \\
\text { Moderate } \uparrow\end{array}$ & $\begin{array}{l}2 \pm 6 \\
\text { Possibly } \\
\text { Small } \uparrow\end{array}$ & & & & & \\
\hline 6 & $\begin{array}{c}29 \pm 11 \\
\text { Most likely } \\
\text { Very large } \uparrow\end{array}$ & $\begin{array}{c}13 \pm 9 \\
\text { Very likely } \\
\text { Very large } \uparrow\end{array}$ & $\begin{array}{r}7 \pm 7 \\
\text { Likely } \\
\text { Large } \uparrow\end{array}$ & $\begin{array}{c}4 \pm 6 \\
\text { Possibly } \\
\text { Moderate } \uparrow\end{array}$ & $\begin{array}{l}2 \pm 6 \\
\text { Possibly } \\
\text { Small } \uparrow\end{array}$ & & & & \\
\hline 7 & $\begin{array}{c}30 \pm 11 \\
\text { Most likely } \\
\text { Very large } \uparrow\end{array}$ & $\begin{array}{c}14 \pm 9 \\
\text { Very likely } \\
\text { Very large } \uparrow\end{array}$ & $\begin{array}{l}8 \pm 7 \\
\text { Likely } \\
\text { Large } \uparrow\end{array}$ & $\begin{array}{c}5 \pm 6 \\
\text { Likely } \\
\text { Moderate } \uparrow\end{array}$ & $\begin{array}{c}3 \pm 6 \\
\text { Possibly } \\
\text { Moderate } \uparrow\end{array}$ & $\begin{array}{c}1 \pm 6 \\
\text { Possibly } \\
\text { Small } \uparrow\end{array}$ & & & \\
\hline 8 & $\begin{array}{c}30 \pm 10 \\
\text { Most likely } \\
\text { Very large } \uparrow\end{array}$ & $\begin{array}{c}14 \pm 9 \\
\text { Very likely } \\
\text { Very large } \uparrow\end{array}$ & $\begin{array}{l}8 \pm 7 \\
\text { Likely } \\
\text { Large } \uparrow\end{array}$ & $\begin{array}{l}5 \pm 6 \\
\text { Likely } \\
\text { Large } \uparrow\end{array}$ & $\begin{array}{c}3 \pm 5 \\
\text { Possibly } \\
\text { Moderate } \uparrow\end{array}$ & $\begin{array}{c}1 \pm 5 \\
\text { Possibly } \\
\text { Small } \uparrow\end{array}$ & $\begin{array}{c}0 \pm 5 \\
\text { Possibly } \\
\text { Trivial } \uparrow\end{array}$ & & \\
\hline 9 & $\begin{array}{c}31 \pm 10 \\
\text { Most likely } \\
\text { Very large } \uparrow\end{array}$ & $\begin{array}{c}15 \pm 9 \\
\text { Very Likely } \\
\text { Very large } \uparrow\end{array}$ & $\begin{array}{c}9 \pm 7 \\
\text { Very likely } \\
\text { Large } \uparrow\end{array}$ & $\begin{array}{l}6 \pm 6 \\
\text { Likely } \\
\text { Large } \uparrow\end{array}$ & $\begin{array}{c}4 \pm 5 \\
\text { Possibly } \\
\text { Moderate } \uparrow\end{array}$ & $\begin{array}{l}2 \pm 5 \\
\text { Possibly } \\
\text { Small } \uparrow\end{array}$ & $\begin{array}{c}1 \pm 5 \\
\text { Possibly } \\
\text { Small } \uparrow\end{array}$ & $\begin{array}{c}1 \pm 4 \\
\text { Possibly } \\
\text { Small } \uparrow\end{array}$ & \\
\hline 10 & $\begin{array}{c}32 \pm 10 \\
\text { Most likely } \\
\text { Very large } \uparrow\end{array}$ & $\begin{array}{c}16 \pm 9 \\
\text { Most likely } \\
\text { Very large } \uparrow\end{array}$ & $\begin{array}{c}10 \pm 7 \\
\text { Very likely } \\
\text { Very large } \uparrow\end{array}$ & $\begin{array}{l}7 \pm 6 \\
\text { Likely } \\
\text { Large } \uparrow\end{array}$ & $\begin{array}{c}5 \pm 5 \\
\text { Likely } \\
\text { Large } \uparrow\end{array}$ & $\begin{array}{c}3 \pm 5 \\
\text { Possibly } \\
\text { Moderate } \uparrow\end{array}$ & $\begin{array}{l}2 \pm 5 \\
\text { Possibly } \\
\text { Small } \uparrow\end{array}$ & $\begin{array}{c}2 \pm 4 \\
\text { Possibly } \\
\text { Moderate } \uparrow\end{array}$ & $\begin{array}{c}1 \pm 4 \\
\text { Possibly } \\
\text { Small } \uparrow\end{array}$ \\
\hline
\end{tabular}

*Data are presented as mean $90 \% \mathrm{Cl}$, likelihood of effect, size and direction of effect $\uparrow$ or $\downarrow$.

$(\mathrm{ES}=0.07)$ to small $(\mathrm{ES}=0.22)$. On the contrary, moderate $(\mathrm{ES}=0.66)$ to very large $(\mathrm{ES}=3.26)$ differences were found when 1- and 2-minute rolling average durations were compared with all other durations (3-10 minutes).

Table 2 reports the pairwise comparisons between rolling average durations and HSR running performance. The distance covered at HSR at 1 minute was very likely to be very large (ES $=2.11-2.58)$ when compared with $2-5$ minute durations. The distances were most likely to be very large $(\mathrm{ES}=2.80-3.26)$ when 1 minute was compared with durations greater than 5 minutes. Further analysis showed that the magnitude of the difference in 2 consecutive rolling average durations decreased as the duration of the rolling average increased.

The pairwise comparisons between rolling average durations and running performance variables for SD are displayed in Table 3. Sprint distance was most likely to be very large (ES $=2.67-4.33$ ) when 1 minute was compared with 3-10 minute rolling average durations. All comparisons among the longest 4 rolling average durations (6-10 minutes) except for 6 vs. 10 minutes $(\mathrm{ES}=0.85$, moderate), 7 vs. 8 minutes $(\mathrm{ES}=0.14$, trivial), and 8 vs. 10 minutes $(\mathrm{ES}=0.67$, moderate) were possibly small $(\mathrm{ES}=0.25-0.34)$.
Figure 1 illustrates the difference in maximal relative TD, HSR, and SD across each time-dependent period. As the time-dependent period decreases relative to the maximal period (10 minutes) there was an increase in maximal relative TD, HSR, and SD covered. There were most likely very large differences between 1- and 2-minute rolling averages compared with the longest window (10 minutes) in TD (ES $=2.04-3.26)$ and HSR $(\mathrm{ES}=2.47-3.35)$. The magnitude of these differences decreased in $\mathrm{TD}(\mathrm{ES}=3.26$, very large to $\mathrm{ES}=0.07$, trivial $), \mathrm{HSR}(\mathrm{ES}=3.35$, very large to $\mathrm{ES}=0.20$, trivial), and $\mathrm{SD}$ ( $\mathrm{ES}=4.33$, very large to $\mathrm{ES}=0.34$, small), as the length of the rolling averages increased.

\section{Running Performance Based on Position}

The maximum relative TD, HSR, and SD were $195 \pm 21$ $\mathrm{m} \cdot \mathrm{min}^{-1}$ (half-backs), $59 \pm 12 \mathrm{~m} \cdot \mathrm{min}^{-1}$ (half-backs), and $45 \pm 9 \mathrm{~m} \cdot \mathrm{min}^{-1}$ (half-forwards), respectively. Descriptive results across all positions for relative TD, HSR, and SD are presented in Table 4 . TD was higher $(p<0.05)$ in half-backs, midfielders, and half-forwards compared with the full-backs and full-forwards. In addition, the half-backs, midfielders, and half-forwards performed higher $(p<0.05)$ 

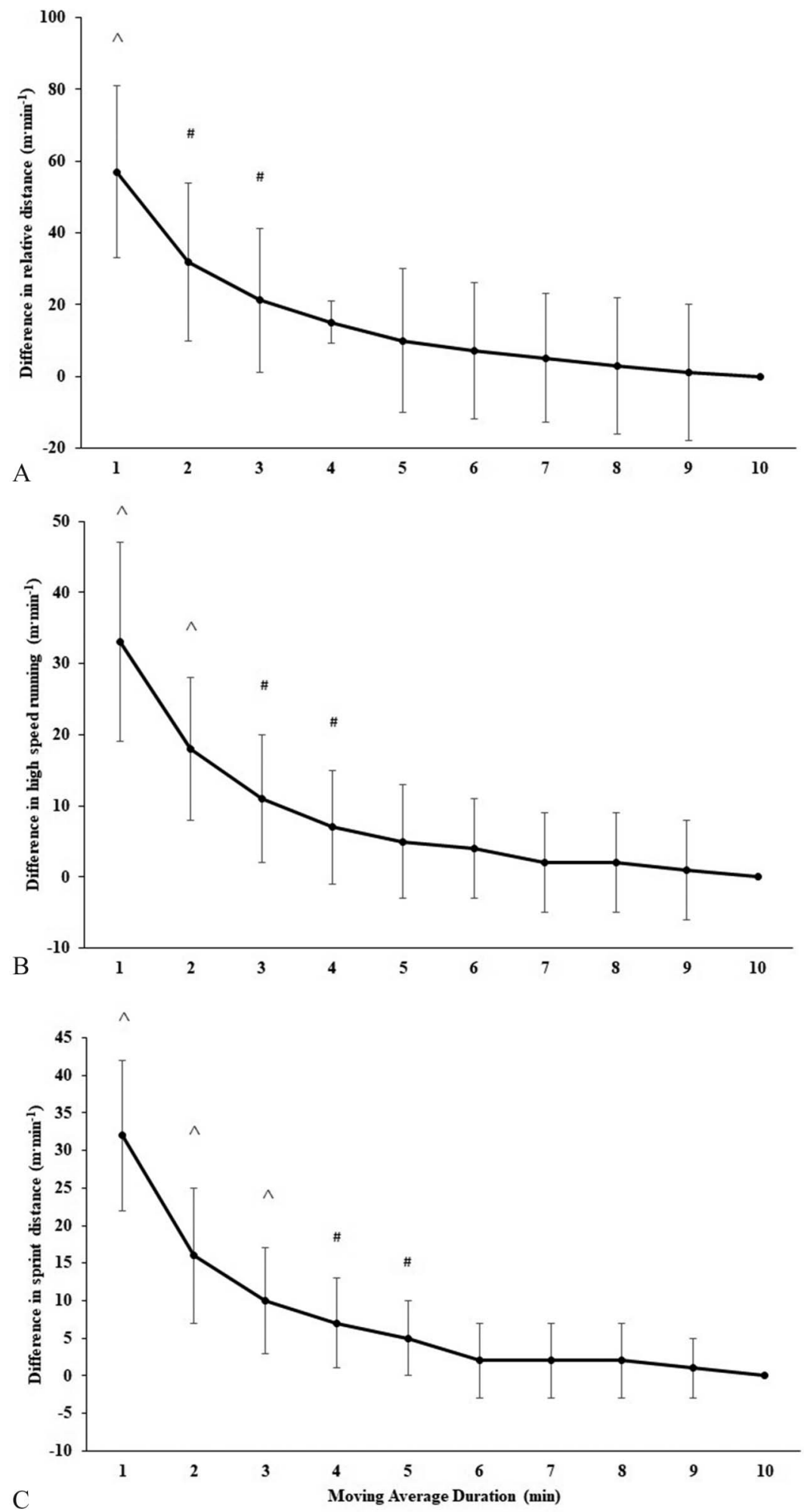

Figure 1. Difference in relative total distance $(\mathrm{A})$, high-speed running distance $(B)$, and sprint distance $(\mathrm{C})\left(\mathrm{m} \cdot \mathrm{min}^{-1}\right)$ compared with 10 -minute rolling average duration. Data are presented as mean $( \pm S D)$. ^ Very large difference from 10 minutes. \#Large difference from 10 minutes.

6 Journal of Strength and Conditioning Research 
TABLE 4. Maximum relative, high-speed running distance and sprint distances $\left(\mathrm{m} \cdot \mathrm{min}^{-1}\right)$ of elite senior hurling players by position for each rolling average duration $( \pm S D)$.

\begin{tabular}{|c|c|c|c|c|c|}
\hline Window length \pm minutes & Full-backs & Half-backs & Midfielders & Half-forwards & Full-forwards \\
\hline \multicolumn{6}{|l|}{ Relative distance $\left(\mathrm{m} \cdot \mathrm{min}^{-1}\right)$} \\
\hline 1 minute & $174 \pm 16$ & $195 \pm 21^{*}$ & $194 \pm 14^{\star}$ & $167 \pm 15^{\star}$ & $167 \pm 15 \dagger \dagger \S$ \\
\hline 2 minutes & $152 \pm 16$ & $168 \pm 13^{*}$ & $171 \pm 11^{*}$ & $166 \pm 16^{*}$ & $142 \pm 13^{*}+\ddagger \S$ \\
\hline 3 minutes & $141 \pm 14$ & $157 \pm 11^{*}$ & $160 \pm 10^{*}$ & $152 \pm 11^{*}$ & $132 \pm 11^{*}+\$ \S$ \\
\hline 4 minutes & $135 \pm 14$ & $150 \pm 9^{*}$ & $153 \pm 12^{*}$ & $146 \pm 10^{*}$ & $126 \pm 13^{*}+\dagger \S$ \\
\hline 5 minutes & $131 \pm 13$ & $146 \pm 8^{*}$ & $147 \pm 9^{*}$ & $143 \pm 10^{*}$ & $121 \pm 13^{\star}+\ddagger \S$ \\
\hline 6 minutes & $129 \pm 11$ & $143 \pm 8^{*}$ & $144 \pm 7^{\star}$ & $138 \pm 10^{*}$ & $118 \pm 12^{\star}+\ddagger \S$ \\
\hline 7 minutes & $126 \pm 12$ & $141 \pm 8^{*}$ & $141 \pm 8^{*}$ & $136 \pm 8^{*}$ & $116 \pm 12^{*}+\S$ \\
\hline 8 minutes & $125 \pm 11$ & $140 \pm 9^{*}$ & $140 \pm 10^{\star}$ & $135 \pm 10^{*}$ & $114 \pm 11^{*}+\ddagger \S$ \\
\hline 9 minutes & $123 \pm 10$ & $137 \pm 9^{*}$ & $137 \pm 8^{*}$ & $133 \pm 9^{*}$ & $112 \pm 11^{*}+\ddagger \S$ \\
\hline 10 minutes & $121 \pm 10$ & $135 \pm 8^{*}$ & $135 \pm 13^{\star}$ & $132 \pm 8^{*}$ & $110 \pm 11^{*}+ \pm \S$ \\
\hline \multicolumn{6}{|c|}{ High-speed running distance $\left(\mathrm{m} \cdot \mathrm{min}^{-1}\right)$} \\
\hline 1 minute & $45 \pm 13$ & $59 \pm 12$ & $53 \pm 13$ & $53 \pm 12^{*}$ & $46 \pm 11 \dagger$ \\
\hline 2 minutes & $31 \pm 9$ & $41 \pm 7^{*}$ & $37 \pm 13^{*}$ & $38 \pm 6^{*}$ & $31 \pm 8 \dagger \dagger$ \\
\hline 3 minutes & $24 \pm 7$ & $33 \pm 5^{\star}$ & $31 \pm 8^{*}$ & $29 \pm 4^{*}$ & $26 \pm 6 \dagger \ddagger$ \\
\hline 4 minutes & $21 \pm 6$ & $29 \pm 6^{\star}$ & $28 \pm 6^{\star}$ & $25 \pm 4^{*} \dagger$ & $22 \pm 6 \dagger+\S$ \\
\hline 5 minutes & $20 \pm 5$ & $27 \pm 6^{*}$ & $25 \pm 5^{\star}$ & $24 \pm 3^{*}$ & $20 \pm 5+\S$ \\
\hline 6 minutes & $18 \pm 5$ & $25 \pm 5^{\star}$ & $23 \pm 5^{\star}$ & $23 \pm 3^{*} \dagger$ & $19 \pm 4 \dagger+\S$ \\
\hline 7 minutes & $17 \pm 5$ & $24 \pm 4^{\star}$ & $22 \pm 4^{\star}$ & $21 \pm 3^{*} \dagger$ & $18 \pm 4 \dagger+\S$ \\
\hline 8 minutes & $17 \pm 5$ & $24 \pm 4^{*}$ & $21 \pm 4^{*}$ & $21 \pm 3^{*} \dagger$ & $17 \pm 4+ \pm \S$ \\
\hline 9 minutes & $16 \pm 5$ & $23 \pm 4^{*}$ & $20 \pm 4^{\star}$ & $20 \pm 3^{*} \dagger$ & $16 \pm 4 \dagger+\S$ \\
\hline 10 minutes & $15 \pm 4$ & $22 \pm 4^{*}$ & $19 \pm 3^{*} \dagger$ & $19 \pm 3^{*} \dagger$ & $16 \pm 4 \dagger+\S$ \\
\hline \multicolumn{6}{|l|}{ Sprint distance $\left(\mathrm{m} \cdot \mathrm{min}^{-1}\right)$} \\
\hline 1 minute & $41 \pm 9$ & $41 \pm 10$ & $43 \pm 12$ & $45 \pm 9$ & $39 \pm 12$ \\
\hline 2 minutes & $24 \pm 5$ & $25 \pm 8$ & $25 \pm 7$ & $25 \pm 11$ & $25 \pm 9$ \\
\hline 3 minutes & $18 \pm 5$ & $20 \pm 6$ & $20 \pm 5$ & $23 \pm 8^{*}$ & $19 \pm 6$ \\
\hline 4 minutes & $15 \pm 4$ & $16 \pm 5$ & $16 \pm 5$ & $19 \pm 5^{*}$ & $16 \pm 5$ \\
\hline 5 minutes & $13 \pm 3$ & $15 \pm 5$ & $14 \pm 4$ & $17 \pm 4^{*}$ & $15 \pm 4$ \\
\hline 6 minutes & $12 \pm 3$ & $13 \pm 5$ & $14 \pm 4$ & $15 \pm 4^{\star}$ & $13 \pm 3$ \\
\hline 7 minutes & $11 \pm 3$ & $12 \pm 4$ & $12 \pm 3$ & $14 \pm 4^{*}$ & $12 \pm 4$ \\
\hline 8 minutes & $11 \pm 3$ & $11 \pm 4$ & $12 \pm 3$ & $13 \pm 4^{*}$ & $11 \pm 3 \S$ \\
\hline 9 minutes & $10 \pm 3$ & $11 \pm 3$ & $11 \pm 3$ & $12 \pm 3^{*} \dagger$ & $11 \pm 3$ \\
\hline 10 minutes & $9 \pm 3$ & $10 \pm 3$ & $10 \pm 2$ & $12 \pm 3^{*} \dagger \ddagger$ & $10 \pm 3$ \\
\hline
\end{tabular}

*Significantly different from $=$ full-backs.

$\dashv$ Significantly different from $=$ half-backs.

$\ddagger$ Significantly different from $=$ midfielders.

$\S$ Significantly different from $=$ half-forwards

HSR compared with the full-backs (1-10 minutes) and fullforwards (4-10 minutes). Except for the 2-minute rolling average, the half-forwards were the highest performers in $\mathrm{SD}$ at each rolling average duration compared with all other positions.

\section{Discussion}

The current study aimed to describe the duration-specific running intensities of elite hurling players during competition with respect to position using a rolling average method. The main findings of the current study were that the matchplay running performance of hurlers was higher than that previously reported (7). Previous research has presented the velocity-based intensity of hurling for the full game and per quarter of the play (7). Although these methods have pro- vided valuable information about the match-play running performances, they under-report the true match-play intensities experienced by players during subtle periods of play, as such our data report for the first time the duration-specific running intensities of hurling match-play. We observed that during match-play, hurlers can attain a relative running intensity of $184 \pm 21 \mathrm{~m} \cdot \mathrm{min}^{-1}$ when compared with the previously reported $109 \pm 17 \mathrm{~m} \cdot \mathrm{min}^{-1}$ (7). Furthermore, depending on the rolling average time period, selected large to very large differences can be observed for relative TD, HSR, and SD. Higher distances were covered in 1-minute; 1- and 2-minute; and 1-, 2-, and 3-minute durations for relative TD, HSR, and SD, respectively, when compared with the 10 -minute rolling average duration. Similar to previous studies in hurling, positional differences were found here 
$(7,26)$. In the current study, half-backs, midfielders, and halfforwards achieved a higher maximal relative TD compared with full-backs and full-forwards. Interestingly, the present results showed that as the time duration increased, the maximum relative TD, HSR, and SD running intensities decreased across all positions.

Previously, the relative running performance for hurlers was reported as $109 \pm 17 \mathrm{~m} \cdot \mathrm{min}^{-1}$ during competitive match-play. The results from the current study show that the maximal relative running intensity was $184 \pm 21$ $\mathrm{m} \cdot \mathrm{min}^{-1}$. The present findings are similar to Gaelic football and highlight that running match-play intensities were underestimated based on previous methodologies used (21). Our data suggest that conditioning for hurlers should include specific periods where these maximal running requirements are attained. However, it must be noted that these values refer to the maximal running performances, which theoretically occur only once during the game. Consequently, conditioning for these "worse-case scenarios" should be part of the overall periodized program (14). The highest relative TD, HSR, and SD values were found in the 1-minute rolling average duration and decreased with each rolling average duration thereafter. The differences in running intensity distance between rolling average durations were small at HSR and SD. For example, for the 3-minute rolling average duration, HSR peak intensity ranged from 4 to $11 \mathrm{~m} \cdot \mathrm{min}^{-1}$. For practical use, differences between rolling average durations were assessed using different thresholds for relative TD, HSR, and SD. These thresholds were selected because it was previously suggested that it would be unlikely that coaches would design training activities so specific that minimal differences in relative distances would need to be identified $(8,21)$. Therefore, these thresholds represent the smallest difference that would have any practical application in the prescription of training content. The analysis showed that the rolling average of 4 to 10 minutes, 5 to 10 minutes, and 6 to 10 minutes in duration for relative TD, HSR, and SD, respectively, produced smaller differences. The rolling average method used in this study was sensitive enough to identify differences between the shorter rolling average windows of a similar duration. This information provides coaches with a valuable structure to facilitate the design of specific hurling training activities, indicating that a change in the duration of just 1 minute in all drills under 4,5 , and 6 minutes could generate large to very large differences in running intensities for TD, HSR, and SD, respectively. Similar findings were observed in the rugby league in which small changes in duration under 6 minutes were proposed to have substantial effects on running performances (8).

The results of the current study were the first to provide position-specific velocity profile for hurling players during competitive match-play. Positional differences were found across rolling averages in relative TD, HSR, and SD. An interesting finding was that as the duration of the rolling average increased, the TD, HSR, and SD continued to decrease for all positions. This reduction in running performance shows that players have difficulty in maintaining the velocity curve maybe due to contextual match-play factors (22). The highest maximal running performances were found during the 1-minute rolling average for all positions. Specifically, full-backs and full-forwards performed the lowest maximal TD and HSR intensities compared with half-backs, midfielders, and half-forwards. These differences in positions may be explained by the differences in specific positional tactical roles. For example, the full-forwards typically are located close to the goal to provide a target to receive possession and try to score. By contrast, the full-backs' role is to remain close to their goal, to man-mark the full-forwards, and to limit the goal scoring chances. However, half-backs, midfielders, and halfforwards have more dynamic tactical roles. They typically tend to move up and down the field depending on where the ball is located. In addition, they retreat toward their own goal when the opposition has possession to prevent scores and move toward the opposition goal in attack to create scoring chances. In addition, half-backs, midfielders, and half-forwards aid the transition of the ball from defense to attack when in possession. Thus, they can accumulate increased running intensities compared with full-backs and full-forwards. Interestingly, fewer differences between positions were observed in maximal SD intensity. As the rolling average duration increased, positional differences appear, with half-forwards completing more SD than full-backs (1-10 minutes), half-backs (9-10 minutes), and midfielders (10 minutes). However, no positional difference in SD was observed during the 1- and 2-minute rolling average durations. It is possible to argue that, although positional differences were significant when distances are presented in meters covered per min, differences ranged between 2 and $5 \mathrm{~m} \cdot \mathrm{min}^{-1}$. Such small differences in SD intensities may not be of practical use for differentiating positions within training sessions. However, these results show that all players need to be able to complete the peak SD intensity (1-minute rolling average) found in the competition.

A limitation of the current study was that only 1 senior hurling team was analyzed. Although the number of subjects used in the present investigation was similar to previously published studies using the rolling average method $(8,21)$, the specific style of play of this team and individual differences in each player's ability to read the game while attacking and defending may have influenced the results. Future research should include additional teams across multiple seasons to provide a holistic representation of match-play running intensities. In addition, the level of opposition, match outcome, and stage of the season was not accounted for, with these known to impact running performances $(13,15,22)$. Future research that compares these variables may provide valuable information on how 
each impact the players' running performance. Finally, because only the maximal distance in each rolling average was analyzed in the current study, adding the number of runs completed would allow coaches to design specific activities to replicate these "worst-case scenarios" in training. Therefore, future research should investigate whether small-sided games or linear drills best replicate these maximal running periods of competition.

\section{Practical Applications}

The current study was the first to describe the durationspecific running intensities of elite hurling players during competition with respect to position using a rolling average method. This methodology provides coaches with valuable information about the players' peak running performance for a range of durations. These results will allow coaches to develop and monitor training activities to ensure that the peak running intensities of match-play can be replicated within different training methodologies. Furthermore, the data provided can be used to identify specific durations, which can be applied to specific training methodologies such as small-sided games while including technical and tactical practice. Practitioners should be aware that increasing the duration of analysis by 1 minute will have large to very large differences on the running intensity in under 4-, 5-, and 6-minute rolling average durations for TD, HSR, and SD, respectively. In addition, the specific positional peak running intensities of competition have been captured and can now be used to ensure that players are sufficiently prepared for the "worst-case scenarios" during match-play. The results of this study showed that the half-backs, midfielders, and half-forwards completed higher peak TD and HSR running intensities compared with full-backs and full-forwards, but no such differences exist for SD. Given the observed data, it would seem prudent to condition the half-backs, midfielders, and half-forwards toward higher TD and HSR when compared with full-backs and full-forwards, whereas all positions can be combined when $\mathrm{SD}$ is the specific target within training.

\section{ACKNOWLEDGMENTS}

The authors thank the players and management of the team who were involved in the current study. The research was funded by grants from the French Ministry of National Education, of Research and of Technology (EA3920) and from Tomsk Polytechnic University Competitiveness Enhancement Program grant, Project No ВИУ-ИСГТ-108/ 2017-TPU CEP-HSTI-108/2017. The authors have no conflicts of interest to disclose.

\section{REFERENCES}

1. Beato, AM, Coratella, G, Schena, F, and Hulton, AT. Evaluation of the external and internal workload in female futsal players. Biol Sport 34: 227-231, 2017.

2. Beato, M, Bartolini, D, Ghia, G, and Zamparo, P. Accuracy of a 10 $\mathrm{Hz}$ GPS unit in measuring shuttle velocity performed at different speeds and distances (5-20 M). J Hum Kinet 54: 15-22, 2016.
3. Beato, M, Impellizzeri, FM, Coratella, G, and Schena, F. Quantification of energy expenditure of recreational football. $J$ Sports Sci 34: 2185-2188, 2016.

4. Bradley, PS, Lago-peñas, C, and Rey, E. Evaluation of the match performances of substitution players in elite soccer. Int J Sports Physiol Perform 9: 415-424, 2014

5. Carling, C and Dupont, G. Are declines in physical performance associated with a reduction in skill-related performance during professional soccer match-play? J Sports Sci 29: 63-71, 2011.

6. Collins, K, Doran, DA, and Reilly, TP. The physiological demands of hurling match-play. In: Contemporary Ergonomics and Human Factors. M. Anderson, ed. CRC Press, London, 2010, pp. 591-595.

7. Collins, K, McRobert, A, Morton, JP, Sullivan, DO, and Doran, DA. The work-rate of elite hurling match-play. $J$ Strength Cond Res 32: 805-811, 2018.

8. Delaney, JA, Scott, TJ, Thornton, HR, Bennett, KJM, Gay, D, Duthie, GM, and et al. Establishing duration-specific running intensities from match-play analysis in rugby league. Int J Sports Physiol Perform 10: 725-731, 2015.

9. Delaney, JA, Thornton, HR, Pryor, JF, Stewart, AM, Dascombe, BJ, and Duthie, GM. Peak running intensity of international rugby: Implications for training prescription. Int J Sports Physiol Perform 12: 1039-1045, 2017.

10. Hopkins, WG. Spreadsheets for analysis for controlled trails with adjustment for a predictor. Sport Sci 10: 46-50, 2006.

11. Jennings, D, Cormack, S, Coutts, AJ, Boyd, LJ, and Aughey, RJ. Variability of GPS units for measuring distance in team sport movements. Int J Sports Physiol Perform 5: 565-569, 2010.

12. Jones, MR, West, DJ, Crewther, BT, Cook, CJ, and Kilduff, LP. Quantifying positional and temporal movement patterns in professional rugby union using global positioning system. Eur $J$ Sport Sci 15: 488-496, 2015.

13. Kempton, $T$ and Coutts, A. Factors affecting exercise intensity in professional rugby league match-play. J Sci Med Sport 19: 504-508, 2016.

14. Lacome, M, Simpson, BM, Cholley, Y, Lambert, P, and Buchheit, M. Small-sided games in elite soccer: Does one side fit all? Int $J$ Sports Physiol Perform 2017. Epub ahead of print: 1-24.

15. Lago, C, Casáis, L, Domínguez, E, Lago, J, and Rey, E. The effect of match location, quality of opposition and match status on work rate in elite soccer. Eur J Hum Mov 23: 107-121, 2010.

16. Maddison, R and Ni Mhurchu, C. Global positioning system: A new opportunity in physical activity measurement. Int J Behav Nutr Phys Act 6: 73, 2009.

17. Malone, S, Collins, KD, and Doran, DA. The running performance and estimated energy cost of hurling specific small-sided games. Int $J$ Sports Sci Coach 11: 853-858, 2016.

18. Malone, S, Roe, M, Doran, DA, Gabbett, T, and Collins, K. Protection against spikes in workload with aerobic fitness and playing experience: The role of the acute: Chronic workload ratio on injury risk in elite Gaelic football. Int J Sport Nutr Exerc Metab 12: 393-401, 2017.

19. Malone, S, Solan, B, Collins, K, and Doran, D. The positional match running performance in elite Gaelic football. J Strength Cond Res 30: 2292-2298, 2016.

20. Malone, S, Solan, B, Collins, K, and Doran, D. The metabolic power and energetic demands of elite Gaelic football match play. J Sport Med Phys Fitness 57: 543-549, 2017.

21. Malone, S, Solan, B, Hughes, B, and Collins, K. Duration specific running performance in elite Gaelic football. J Strength Cond Res, 2017. Epub ahead of print.

22. Mangan, S, Malone, S, Ryan, M, McGahan, J, O’Neill, C, Burns, C, and et al. The influence of match outcome on running performance in elite Gaelic football. Sci Med Footb 2017. Epub ahead of print. 
23. Mohr, M, Krustrup, P, and Bangsbo, J. Match performance of highstandard soccer players with special reference to development of fatigue. J Sports Sci 21: 519-528, 2003.

24. Reilly, T and Collins, K. Science and the Gaelic sports: Gaelic football and hurling. Eur J Sport Sci 8: 231-240, 2008.

25. Varley, MC, Elias, GP, and Aughey, RJ. Current matchanalysis techniques' underestimation of intense periods of high-velocity running. Int J Sport Physiol Perform 7: 183-185, 2012.

26. Young, D, Mourot, L, Beato, M, and Coratella, G. The match heartrate and running profile of elite under 21 hurlers during competitive match-play. J Strength Cond Res 2018. Epub ahead of print.

27. Young, D, Mourot, L, and Coratella, G. Match-play performance comparisons between elite and sub-elite hurling players. Sport $S c i$ Health 14: 201-208, 2018. 